\title{
Primary Cutaneous Aspergillosis in an Immunocompetent Patient: A Case Report from a Tertiary Care Hospital in Chennai
}

\author{
Sathyakamala Ravichandran ${ }^{1}$ Priyadarshini Shanmugam ${ }^{1} \quad$ Ambujavalli Balakrishnan Thayikkannu ${ }^{1}$ \\ Pradeep Elangovan ${ }^{2}$ \\ ${ }^{1}$ Department of Microbiology, Chettinad Hospital and Research \\ Institute, Chettinad Academy of Research and Education, \\ Kelambakkam, Tamil Nadu, India \\ 2 Department of Orthopaedics, Chettinad Hospital and Research \\ Institute, Chettinad Academy of Research and Education, \\ Address for correspondence Priyadarshini Shanmugam, MD, PhD, \\ Department of Microbiology, Chettinad Hospital and Research \\ Institute, Chettinad Academy of Research and Education, \\ Kelambakkam, 603103, Tamil Nadu, India \\ (e-mail: priyadarshini0018@gmail.com).
} Kelambakkam, Tamil Nadu, India

J Lab Physicians 2022;14:355-361.

\begin{abstract}
Keywords

- Aspergillus flavus

- immunocompetent

- primary cutaneous aspergillosis

- systemic fungal infection

Aspergillosis is a systemic fungal infection that commonly affects immunocompromised individuals and, less frequently, immunocompetent individuals. It is the most common opportunistic fungal disease after candidiasis. This is primarily a pulmonary infection and can also involve other body sites like paranasal sinuses and cutaneous tissues. Aspergillus fumigatus, Aspergillus niger, and Aspergillus flavus are the common species infecting humans. Primary cutaneous aspergillosis (PCA) is usually caused by $A$. flavus and $A$. fumigatus. It is commonly seen in immunocompromised patients such as those suffering from diabetes, malignancies, tuberculosis, human immunodeficiency virus, or patients on long-term steroids and antibiotics. In this article, we report a case of PCA, in the immediate postoperative period, following a road traffic accident, in an immunocompetent patient. This posed a diagnostic challenge to the treating physicians. A. flavus was confirmed with $10 \%$ potassium hydroxide mount, lactophenol cotton blue, and growth on Sabouraud dextrose agar from tissue culture sample. Antifungal treatment was initiated with oral itraconazole $200 \mathrm{mg}$ after performing antifungal susceptibility testing based on Clinical and Laboratory Standards Institute guidelines. The patient's condition improved and was discharged. Thus, early detection of PCA combined with medical and surgical intervention can successfully eradicate infection and help in preventing disseminated aspergillosis.
\end{abstract}

\section{Introduction}

Aspergillosis is a systemic fungal infection that commonly affects immunocompromised individuals and less frequently immunocompetent individuals. It is the commonest opportunistic fungal disease after Candidiasis. ${ }^{1}$ This is primarily a pulmonary infection and can also involve other body sites, like para nasal sinuses and cutaneous tissues. It is caused by published online February 15, 2022
DOI https://doi.org/

$10.1055 / \mathrm{s}-0042-1742633$. ISSN 0974-2727. (c) 2022. The Indian Association of Laboratory Physicians. All rights reserved.

This is an open access article published by Thieme under the terms of the Creative Commons Attribution-NonDerivative-NonCommercial-License, permitting copying and reproduction so long as the original work is given appropriate credit. Contents may not be used for commercial purposes, or adapted, remixed, transformed or built upon. (https://creativecommons.org/ licenses/by-nc-nd/4.0/)

Thieme Medical and Scientific Publishers Pvt. Ltd., A-12, 2nd Floor, Sector 2, Noida-201301 UP, India 
several species of genus Aspergillus, which are predominantly found in the environment, in decaying organic matter. ${ }^{2}$

Aspergillus fumigatus, Aspergillus niger, and Aspergillus flavus are the common species infecting humans. Primary cutaneous aspergillosis (PCA) is usually caused by A. flavus and $A$. fumigatus. ${ }^{3}$ It is commonly seen in patients with compromised immune system such as those suffering from diabetes, chronic diseases like tuberculosis, those on longterm steroids and antibiotics, patients with malignancies on immunosuppressive therapy, and human immunodeficiency virus. Organ transplant and autoimmune disease also increase the risk. ${ }^{4}$ Patients of PCA can present with molluscum like papules, nodules, ulcers, and plaques. ${ }^{1}$

In this article, a case of PCA, in the immediate postoperative period, following a road traffic accident, in an immunocompetent patient is reported. This posed a diagnostic challenge to the treating physicians.

\section{Case Report}

A 50-year-old female patient presented with multiple phalangeal fracture and second metatarsal undisplaced fracture of the left foot caused by a motorcycle accident ( - Fig. 1). She had no other comorbidities. Her blood sugar levels were normal, was normotensive, and was not on steroids. Her renal parameters were normal and she did not have any previous history of antifungal use. All other blood investigations performed were within the normal range. Fracture treatment included Kirschner wire fixation (-Fig. 2). Repeated surgical debridement of the wound was also done since it had large tissue defects (-Figs. 3 and 4). No samples were sent for culture at this point of time. There was no intensive care unit admission during this period. The patient was discharged with the advice to report regularly for wound dressing on alternate days, for which the patient did not comply. A week later, the patient reported to the outpatient department with pain and on examination, the wound looked unhealthy. She was, therefore, admitted for wound debridement and regular saline dressing. Her wound was dressed regularly and tissue bit was sent for culture in view of presence of necrotic tissue suggestive of infection. Ten percent potassium hydroxide $(\mathrm{KOH})$ mount culture and lactophenol cotton blue (LPCB) mount were performed. KOH mount showed hyaline septate hyphae. Light green velvety colonies of A. flavus was isolated in culture (-Figs. 5,6,7,8,9). LPCB mount demonstrated conidiophores of variable length, rough, pitted and spiny along with biseriate phialides covering the entire vesicle and pointing out in all directions (-Figs. 10,11,12).

Additional two tissue bit samples were obtained on consecutive days. This helped to distinguish between colonization and infection. $\mathrm{KOH}$ mount was positive for septate hyphae and the growth was similar to previously reported cultures. Antifungal susceptibility testing was performed

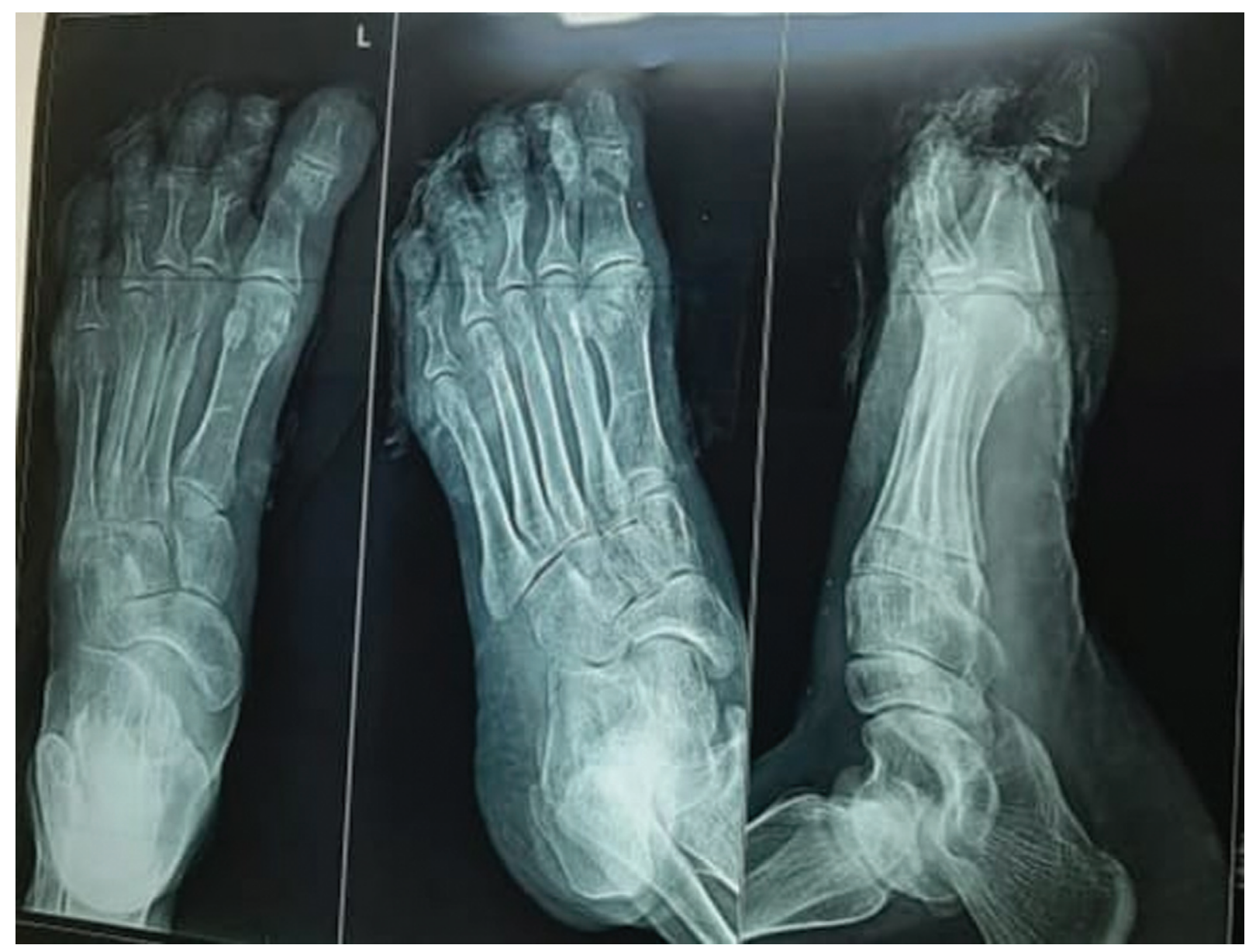

Fig. 1 Preoperative X-ray image showing multiple phalangeal fracture and second metatarsal undisplaced fracture of the left foot. 


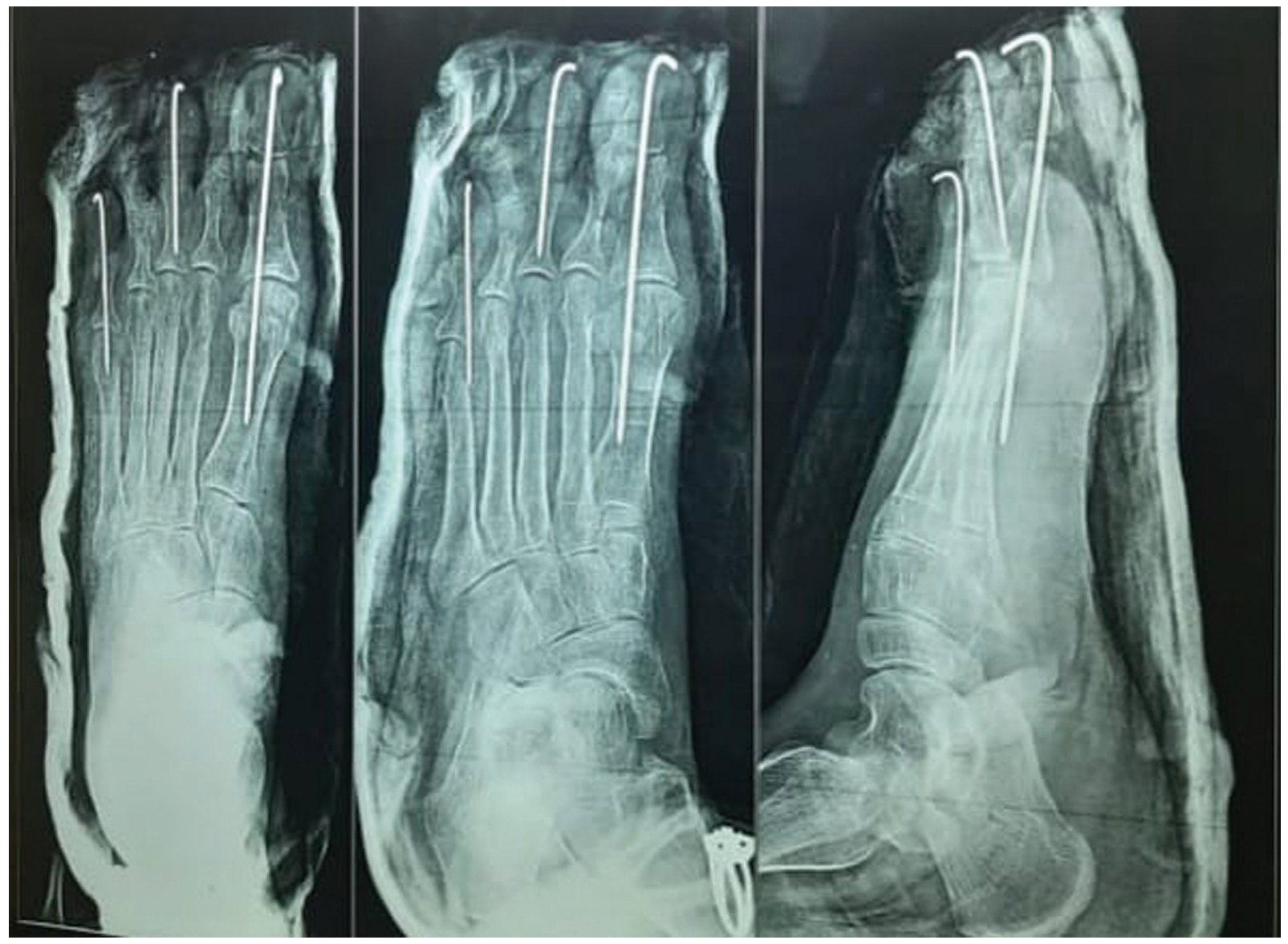

Fig. 2 Postoperative X-ray image showing surgery done with Kirschner wire fixation.

according to Clinical and Laboratory Standards Institute guidelines (-Fig. 13). It showed resistance to fluconazole, ketoconazole, amphotericin-B, and nystatin and was sensitive to clotrimazole, voriconazole, and itraconazole. Infectious necrotic tissue areas persisted despite surgical debridement. Further, A. flavus growth was observed in all three consecutive cultures. Hence, antifungal treatment was initiated and patient was started on oral itraconazole $200 \mathrm{mg}$ twice daily for 10 days.

There was no other factor contributing to immunocompromised status of the patient. There were no findings suggestive of onychomycosis. No other bacterial organism was isolated from the samples sent. All three cultures yielded A. flavus proving it to be a true pathogen.

Regular saline dressing on a daily basis and surgical debridement were continued. Tissue bit cultures sent after the end of antifungal therapy yielded no growth. Saline dressing was continued till the wound healed (-Fig. 14). Patient's condition improved. She was then discharged on oral analgesics (SOS) alone. She was advised to come regularly on a weekly basis for change of dressing.

\section{Discussion}

A. flavus, an important causative agent of invasive aspergillosis, can also commonly cause superficial infections, even of those affecting the skin and oral mucosa. ${ }^{5}$ A. flavus grows optimally at $37^{\circ} \mathrm{C}$. Growth is also observed at temperatures ranging from 12 to $48^{\circ} \mathrm{C}^{6}$ This is an important reason contributing to its pathogenicity in humans.

The cutaneous infections are generally classified as primary and secondary. Primary infection can be triggered by several causes including direct instillation, like traumatic inoculation, intravenous catheters, occlusive dressings, burns, and surgical procedures. Secondary infection happens mostly from hematogenous spread. ${ }^{7}$

In our case, A. flavus growth was seen in an immunocompetent patient following a road traffic accident. All other parameters were normal, the only source of infection could be traumatic inoculation. The patient reported to the emergency department initially with acute trauma, following which she was operated and managed by the orthopaedic team. No samples were sent for culture at this point of time, as per the protocol, keeping in mind the early surgical treatment needed for the patient. After surgery and discharge, patient was asked to come for regular dressings on alternate days. But the patient reported only after a week, with unhealthy looking superficial wound and complaints of pain. The wound being superficial rules out the possibility of an iatrogenic inoculation. The possible ways by which healthy hosts can develop cutaneous aspergillosis are traumatic inoculation, surgical wound operations, or exposure to high levels of sporulating organisms in occupations such as farming. This patient had no history of farming activities. A 
358 Cutaneous Aspergillus flavus Infection in an Immunocompetent Patient Ravichandran et al.

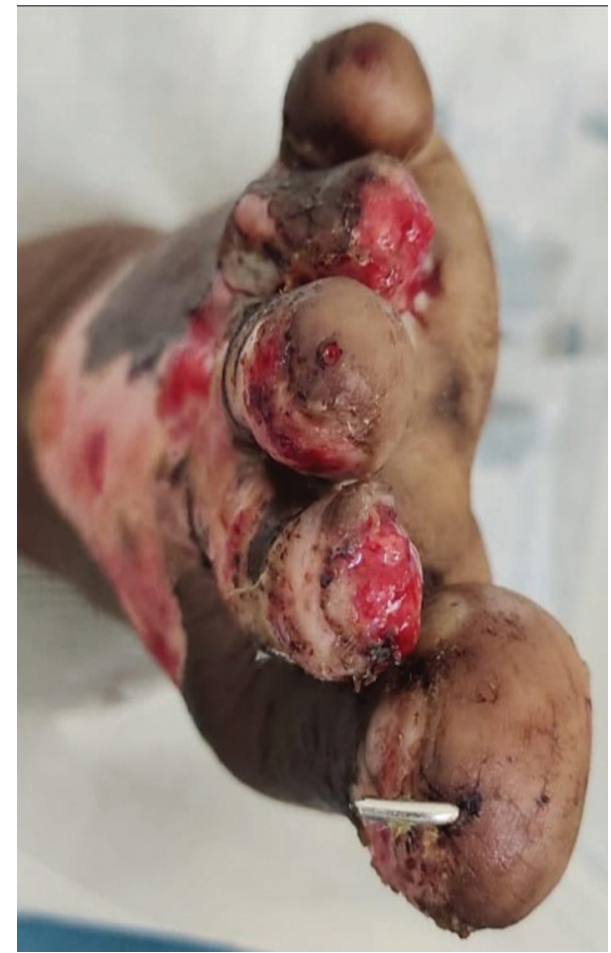

Fig. 3 Preoperative image showing gross injury and tissue damage with necrosis.

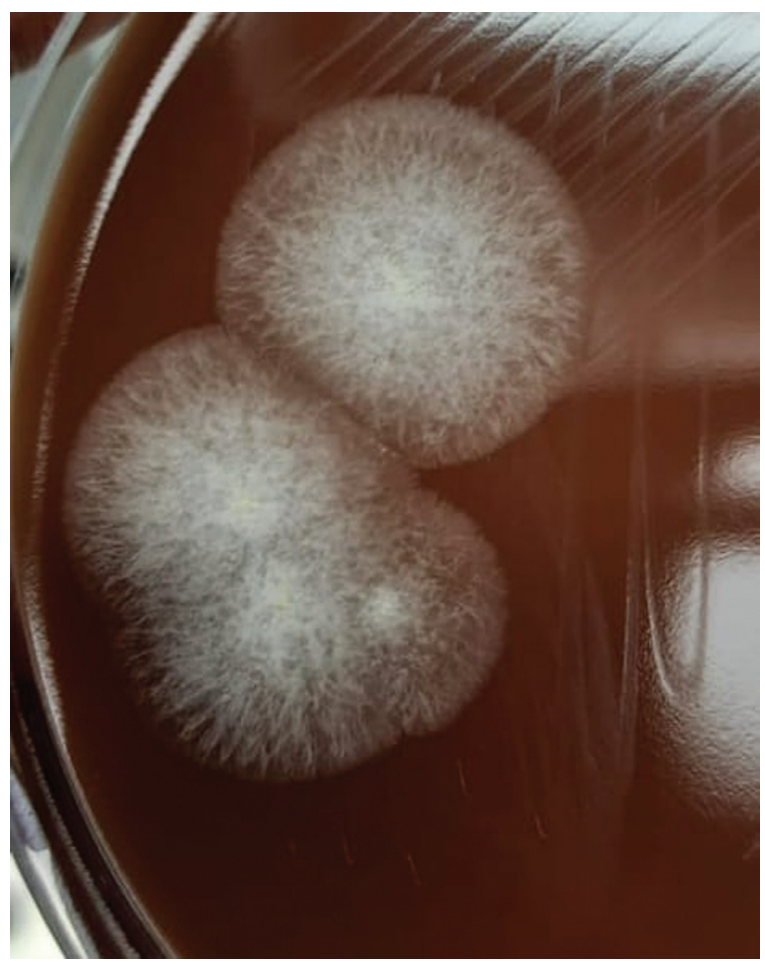

Fig. 5 Initial moldy growth observed on chocolate agar.

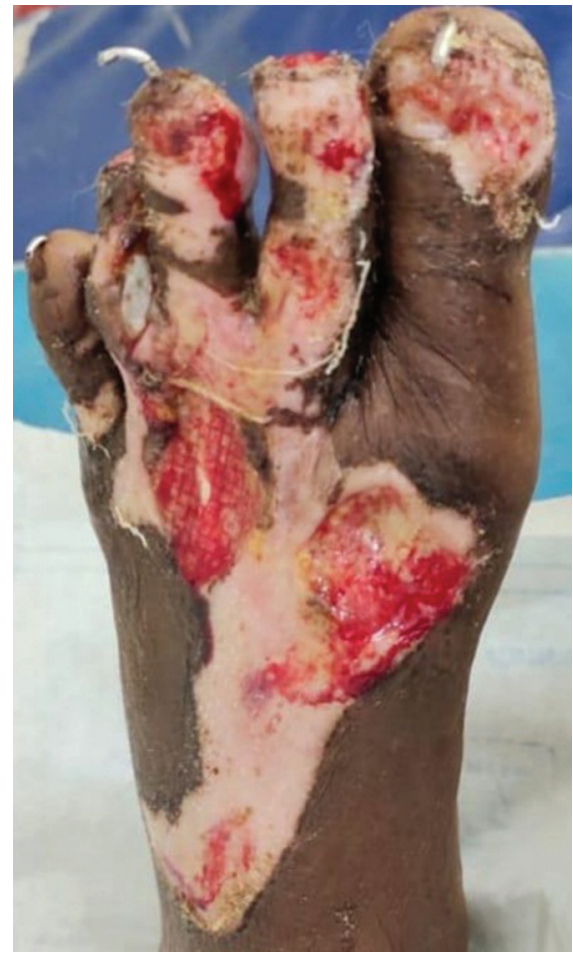

Fig. 4 Preoperative picture showing extensive tissue damage and necrosis of foot.

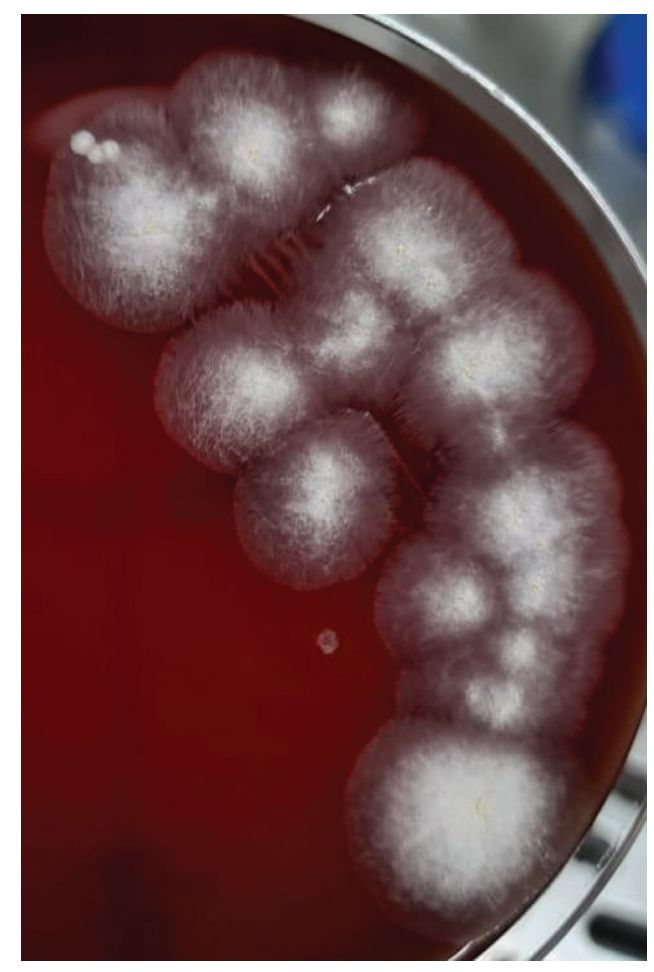

Fig. 6 Initial mouldy growth observed on blood agar. 


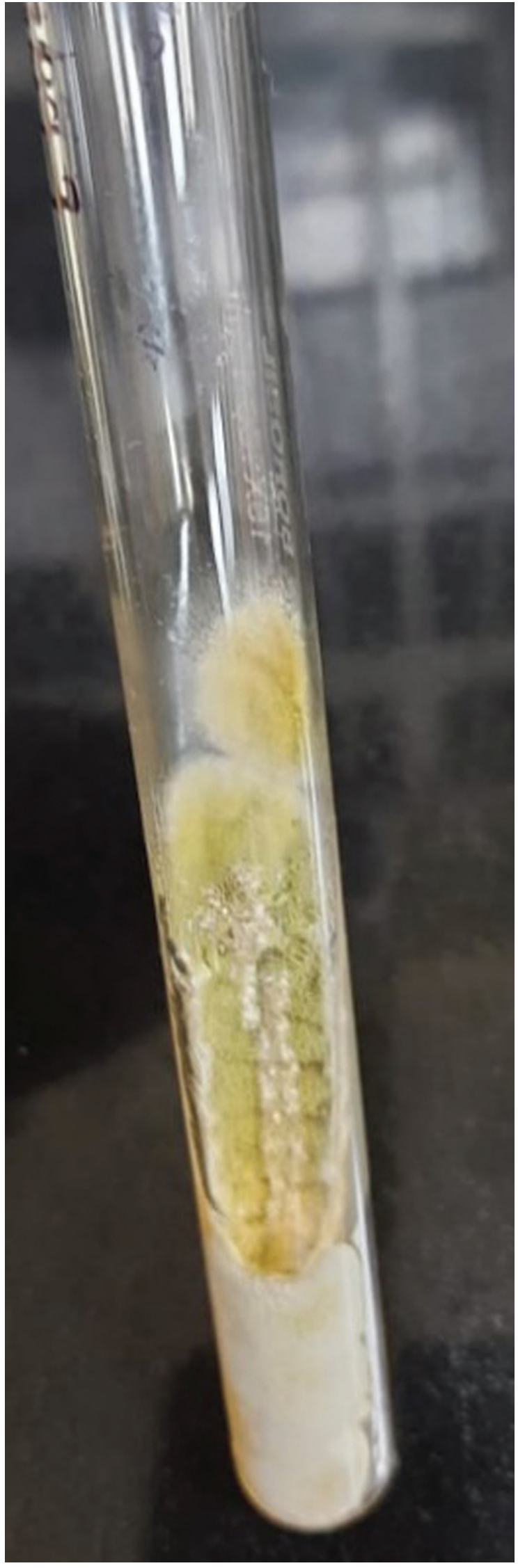

Fig. 7 SDA slope showing yellowish-green mycelial colonies suggestive of Aspergillus flavus.

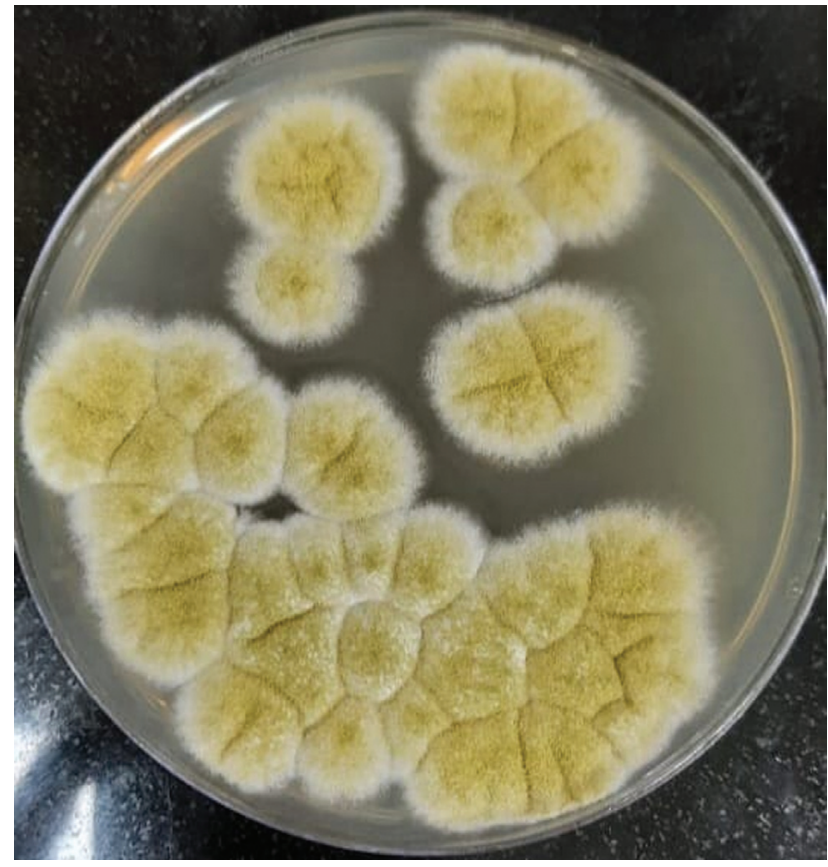

Fig. 8 SDA agar showing yellowish-green mycelial colonies suggestive of Aspergillus flavus, obtained from tissue bit culture.

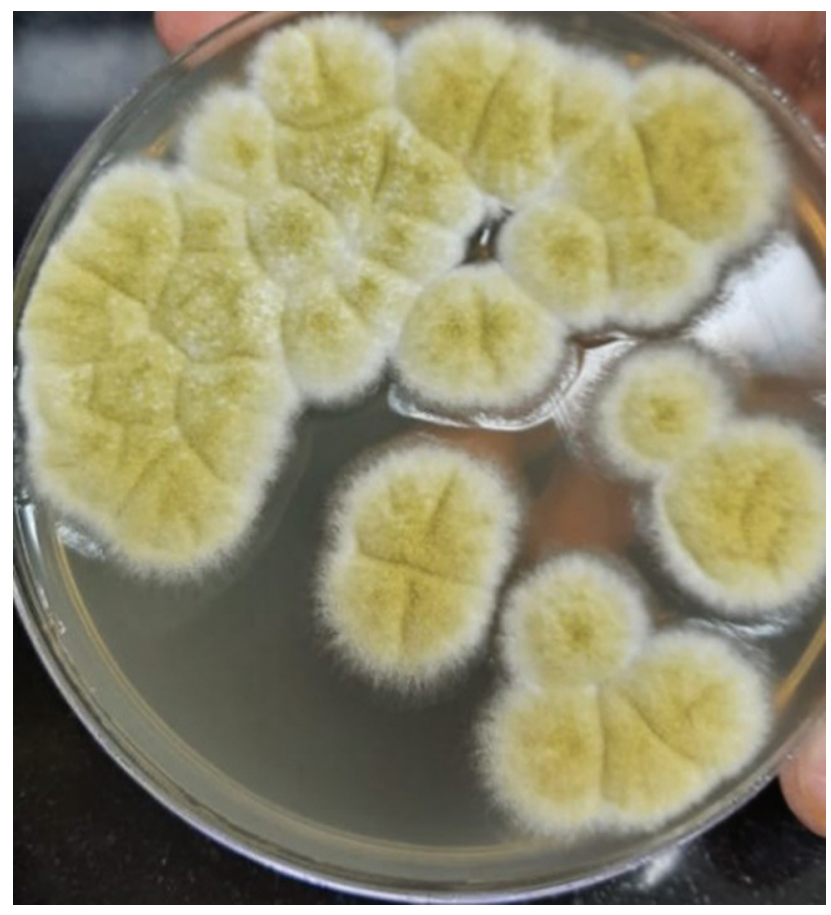

Fig. 9 SDA agar showing yellowish-green mycelial colonies suggestive of Aspergillus flavus, obtained from repeat tissue bit culture for confirmation.

similar growth of Aspergillus spps. has been noted in immunocompetent patients in the studies of Tahir et al, Camus et al, Avkan-Oğuz et al, and Neki et al. ${ }^{2,8-10}$

The treatment of PCA in immunocompromised patients is well understood but the management of PCA in immunocompetent patients is less comprehensible and poses a challenge to the treating physicians. ${ }^{11}$ In our case, the patient 


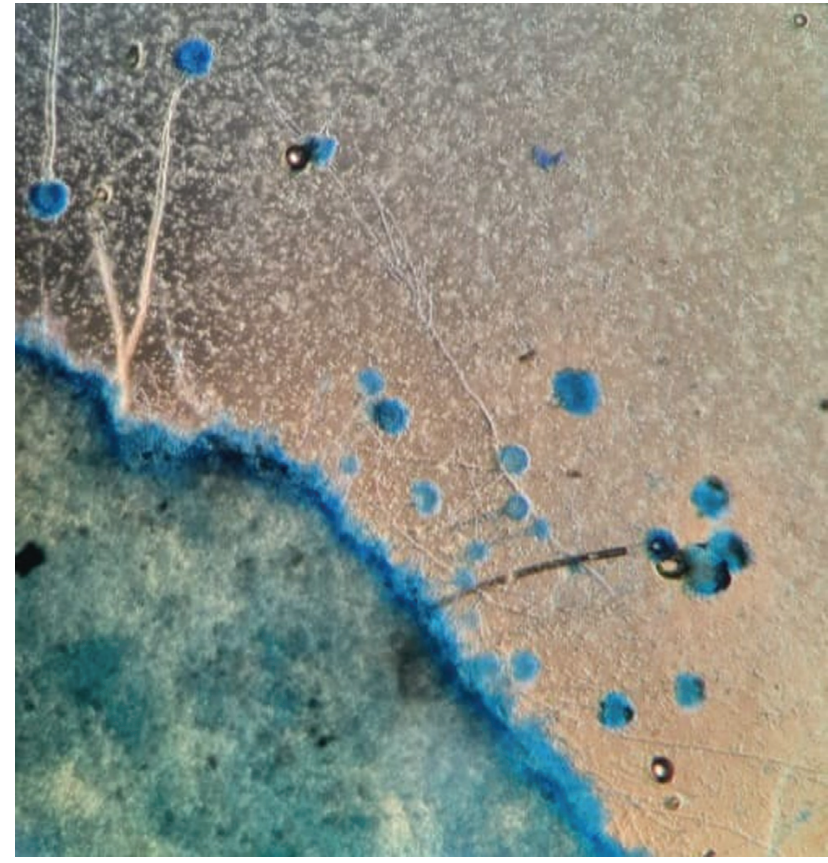

Fig. 10 LPCB mount showing conidia, vesicles, conidiophores, and septate hyphae suggestive of Aspergillus flavus, under low power magnification.

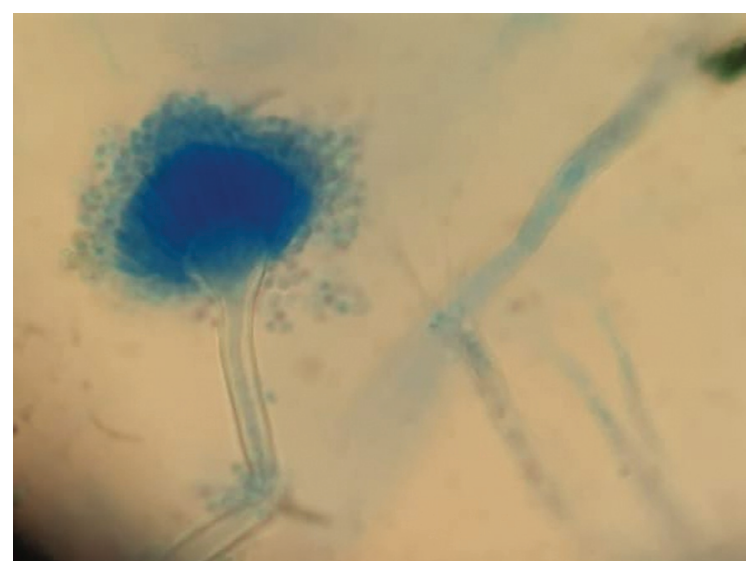

Fig. 11 LPCB mount showing conidia, vesicles, and conidiophores of Aspergillus flavus, under high power magnification, taken from sample sent for tissue bit culture.

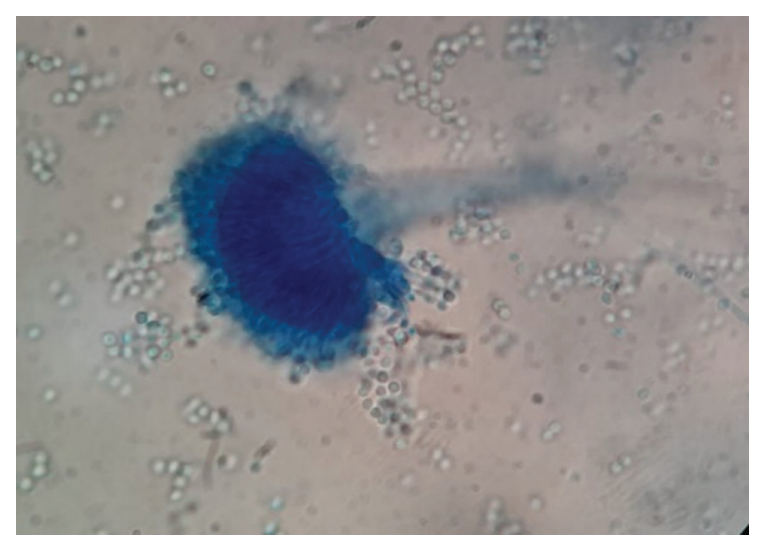

Fig. 12 LPCB mount showing conidia, vesicles, and conidiophores of Aspergillus flavus, under high power magnification, taken from sample sent for repeat tissue bit culture.

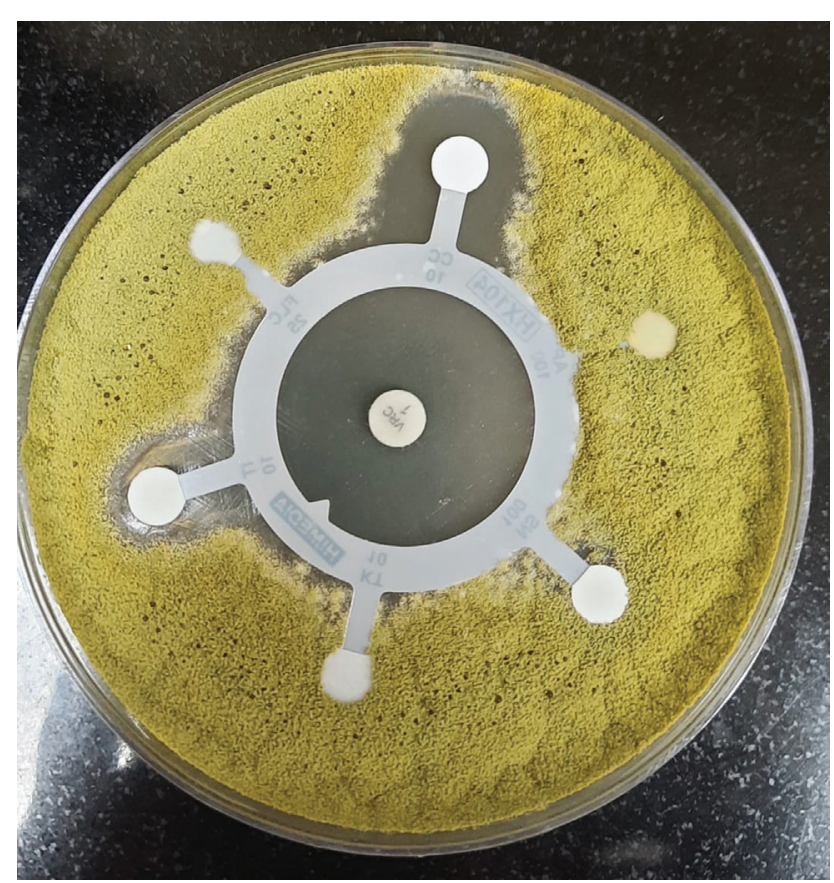

Fig. 13 Antifungal susceptibility test performed, showing resistance to multiple drugs, including Amphotericin-B and Nystatin.

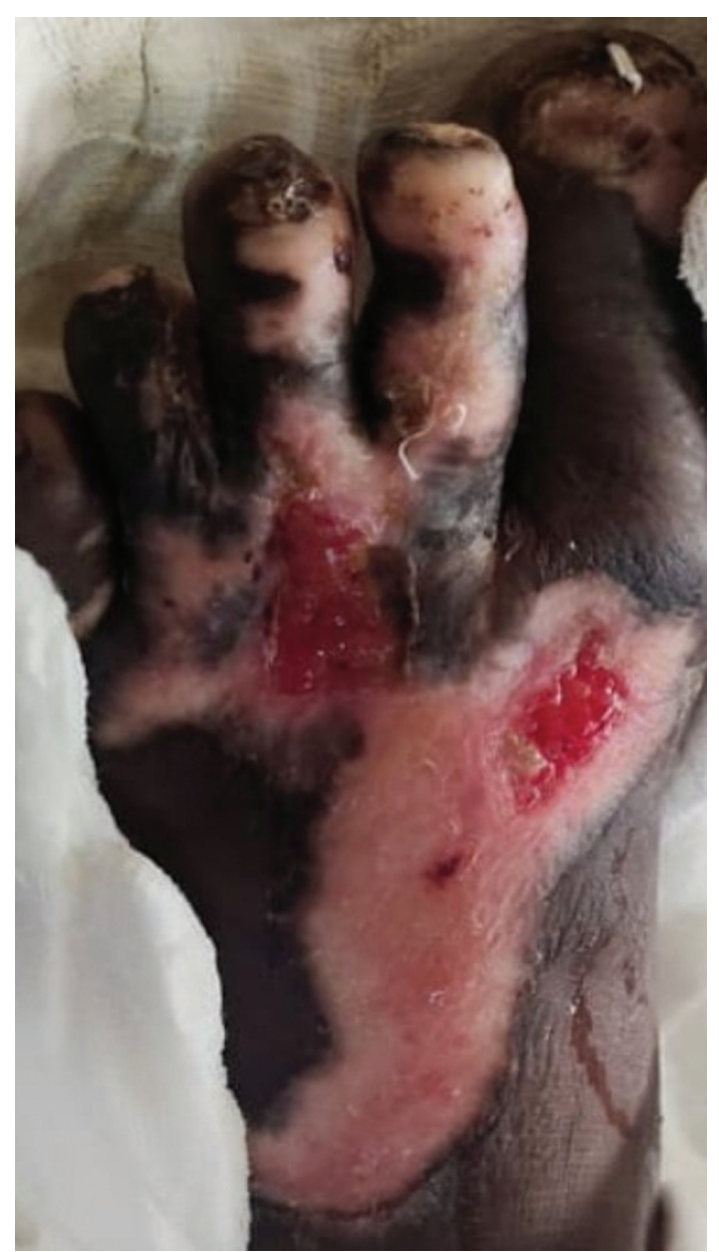

Fig. 14 Postoperative picture showing healed areas with improved tissue function, taken after surgical debridement and antifungal therapy. 
responded very well to oral antifungal therapy with itraconazole. She showed no adverse side effects and improved well. Amphotericin B is the drug of choice for disseminated aspergillosis. Oral itraconazole, a triazole antifungal with activity against Aspergillus species, is an alternative to amphotericin B for all types of aspergillosis. Voriconazole is given for the most advanced cases. ${ }^{12}$

Thus, there has been limited case reports on aspergillosis reported in immunocompetent patients. The accurate identification and appropriate treatment give better wound healing and prognosis. The treatment of cutaneous aspergillosis causes no harm to healthcare workers who are immunocompetent. ${ }^{13}$ Early detection, combined with medical and surgical intervention, can successfully eradicate infection. This will help in treating the locally destructive disease and thereby prevents disseminated aspergillosis. ${ }^{14}$

\section{Conclusion}

To conclude, the most beneficial management plan would be to make decisions based on individual history and clinical findings. Appropriate antifungal treatment combined with regular dressing and repeated surgical debridement till culture negativity should be followed. ${ }^{15}$

\section{Authors' Contribution}

SR, $A B$, and PS collected and analyzed the data. SR and AB prepared the manuscript and it was reviewed and modifications were made by PS. SR participated in the manuscript revision under the guidance of $A B$ and PS. All authors of the manuscript gave their complete approval.

Funding

None.

Conflict of Interest

None declared.

\section{References}

1 Chander J. Textbook of Medical Mycology. 4th edition. New Delhi: Jaypee Brothers; 2018:524-553
2 Tahir C, Garbati M, Nggada HA, Yawe EH, Abubakar AM. Primary cutaneous aspergillosis in an immunocompetent patient. J Surg Tech Case Rep 2011;3(02):94-96

3 Bagate A, Birare S, Jadhav DS, Nalpe V, Patekar D. Cutaneous aspergillosis in an immunocompetent patient: a case report. Tropical Journal of Pathology and Microbiology 2020;6(02): 205-209. Doi: 10.17511/jopm.2020.i02.14

4 Zhang T, Christopher M, Simske NM, Saddler CM, Keenan T, Whiting PS. Fracture treatment in the setting of cutaneous aspergillosis: a case report. OTA Int 2020;3(03):e082. Doi: 10.1097/OI9.0000000000000082. PMID: 33937705; PMCID: PMC8023117

5 Khatri ML, Stefanato CM, Benghazeil M, Shafi M, Kubba A, Bhawan J. Cutaneous and paranasal aspergillosis in an immunocompetent patient. Int J Dermatol 2000;39(11):853-856

6 Mohapatra S, Xess I, Swetha JV, et al. Primary cutaneous aspergillosis due to Aspergillus niger in an immunocompetent patient. Indian J Med Microbiol 2009;27(04):367-370

7 Chakrabarti A, Gupta V, Biswas G, Kumar B, Sakhuja VK. Primary cutaneous aspergillosis: our experience in 10 years. J Infect 1998; 37(01):24-27

8 Camus M, Anyfantakis V, Dammak A, Riche A, Delouane H, Roblot F, Guillet G. Aspergillose cutanée primitive chez un agriculteur immunocompétent [Primary cutaneous aspergillosis in an immunocompetent farmworker]. Ann Dermatol Venereol 2010;137 (05):373-376. Doi: 10.1016/j.annder.2010.03.006 Epub 2010 Apr 24. PMID: 20470919.

9 Avkan-Oğuz V, Çelik M, Satoglu IS, Ergon MC, Açan AE. Primary cutaneous Aspergillosis in immunocompetent adults: three cases and a review of the literature. Cureus 2020;12(01):e6600. Doi: $10.7759 /$ cureus.6600

10 Neki N, Singh A, Shergill G, Sidhu P, Pannu J, Singh T. Cutaneous Aspergillosis in an immunocompetent patient. Int J Med Health Res 2016;2:57-58

11 Sharma S, Yenigalla BM, Naidu SK, Pidakala P. Primary cutaneous aspergillosis due to Aspergillus tamarii in an immunocompetent host. BMJ Case Rep 2013;2013:010128. Doi: 10.1136/bcr-2013010128

12 Chong YS, Ro BI. Cutaneous aspergillosis. Korean J Med Mycol 2002;7(01):6-13

13 Granstein RD, First LR, Sober AJ. Primary cutaneous aspergillosis in a premature neonate. Br J Dermatol 1980;103(06):681-684

14 Venugopal TV, Venugopal PV. Primary cutaneous aspergillosis from Tamil Nadu diagnosed by fine needle aspiration cytology. Med Mycol Case Rep 2012;1(01):103-106

15 Liu X, Yang J, Ma W. Primary cutaneous aspergillosis caused by Aspergillus fumigatus in an immunocompetent patient: a case report. Medicine (Baltimore) 2017;96(48):e8916. Doi: 10.1097/ MD.0000000000008916 